\title{
On the parametrization of clapping
}

\author{
Herwin van Welbergen and Zsófia Ruttkay \\ Human Media Interaction, University of Twente Enschede, The Netherlands \\ $\{$ welberge, ruttkay\}@ewi.utwente.nl
}

\section{Introduction}

Recently we have been interested in creating embodied conversational agents (ECAs) with believable expressive verbal and nonverbal behavior. One of the applications is the RVT: an ECA in the role of a real physiotherapist, capable of 'acting out' the exercise sequences the user is supposed to do. This virtual trainer is reactive: she perceives the performance of the user, and adapts her reactions accordingly. The reaction often involves change of tempo of the exercises to be performed. In such exercises subtle timing and lifelikeness of the motion is of primary importance: we want to be sure that the user mimics the motion of the RVT to the detail, otherwise it may not achieve the envisioned positive effect, or may be even harmful. The RVT - similarly to real trainers - accompanies some of the exercises with counting, to emphasize the required tempo. In this paper we address the issue of change of tempo of rhythmic motion, particularly exercises involving the movement of the arms and hands, like clapping. Our goal is to find the consequences of a change of the tempo on other characteristics of the motion in order to be able to generate (physiotherapy) exercises. We discus subtle characteristics and synchronization and timing strategies discovered in analysis of clapping of two subjects.

Analysis of rhythmic limb motion has been done in biomechanical research. However, such research is typically performed in very constrained environment in which one movement characteristic is tested extensively. We do our measurements in a un-obtrusive environment and aim to obtain insight on a wider range of characteristics, on an abstraction suitable to be used as control parameters in motion generation. With the result we have found, we plan to extend existing work on model based gesture synthesis with whole body involvement, variability and parameterization.

\section{Setup of the experiments}

Two subjects were first asked to clap and to count from 21 to 30 in while clapping, further called 'free clapping'. Then, the subjects were asked to clap to the beat of a metronome and to count while clapping, further called 'metronome driven clapping'. The metronome is set at 30, 50, 70, 90, 110, 150, 180, 210 and 240 beats per minute. The subject had to clap twelve times at each tempo, after which the tempo was increased. The subjects were recorded using both optical motion capture and video camera. 


\section{Results}

Inspired by the movement phases identified in gestures [2], we decompose a single clap into four phases. The hands can be held in their starting position in an pre-stroke hold phase. During the stroke phase, the hands move together. The hands can then be held together in an optional post-stroke hold phase. In the retraction phase, the hands move back to their initial positions. The phonological synchrony rule states that the moment of peak gesture effort (in the stroke phase) of a gesture precedes or ends at the phonological peak syllable of the speech. For both subjects this rule was validated.

Our experiments have shown that clapping movement is often sped up just by making the path distance shorter, keeping the average speed and the relative timing of clapping phases the same. If this speedup strategy is used, the path distance decreases linearly with the clapping speed. The average movement speed on the path is quite constant and does not change with the metronome tempo. The value of this speed is depended on the movement phase (retraction or stroke) and on the subject (or his/her clapping style), but it does not vary much between hands. At $30 \mathrm{bpm}$, one of the subjects made use of a pre-stroke as a slowdown strategy.

Involvement of the whole body is crucial to make an animation believable [1]. We found that clapping is clearly a whole body motion. Movement related to the clap is perceived on the head, torso and even down to the knees. At a higher tempo, fewer body parts are perceivably involved.

The stability of the symmetry of rhythmically moving limbs depends on the mass imbalance between them and the clapping frequency [3]. We found that this was valid for rhythmic clapping as well: the standard deviation of the phase difference between hands, $\phi$, increased with the clapping frequency. We observed no significant increase of the mean of $\phi$ with the clapping frequency. In an inphase finger tapping task, it has been shown that right-handed individuals lead the tapping with their right finger [3]. However, such asymmetry often disappears when the task is constrained to a metronome. Both our right handed subjects lead the clapping with their right hand. The metronome did not seem to effect the mean $\phi$ : similar mean $\phi$ values were found for free clapping and metronome driven clapping.

\section{References}

1. Norman I. Badler, Monica Costa, Liwei Zhao, and Diane M. Chi. To gesture or not to gesture: What is the question? In Proceedings of the International Conference on Computer Graphics, pages 3-9, Washington, 2000. IEEE Computer Society.

2. D. McNeill. Hand and Mind: What Gestures Reveal about Thought. University of Chicago Press, Chicago, 1995. ISBN 0-226-56134-8.

3. P. J. Treffner and M. T. Turvey. Symmetry, broken symmetry, and handedness in bimanual coordination dynamics. Experimental Brain Research, 107(3):463-478, January 1996. 